\title{
sciendo
}

DOI: 10.1515/aon-2019-0008

\section{SBAS GUIDELINES FOR SHIPBORNE RECEIVER: EGNOS PERFORMANCE BASED ON IMO RES. A.1046 (27)}

\author{
Sergio Magdaleno (ESSP) \\ Elisabet Lacarra (ESSP) \\ Carlos de la Casa (ESSP) \\ Manuel López (GSA) \\ Roberto Roldán (ESSP) \\ Nuria Blanco (ESSP)
}

\begin{abstract}
The European Geostationary Navigation Overlay Service (EGNOS) augments the open public service offered by the GPS in Europe making suitable the use of GPS for safety critical applications. EGNOS is designed according to the same standard [ICAO SARPs, 2018] such as US WAAS, Japanese MSAS, GAGAN in India, SDCM in Russia and KAAS in South Korea and provides over Europe both corrections and integrity information about the GPS system. As the European SBAS, EGNOS offers three services: Open Service, Safetyof-life Service and EDAS. In general, the EGNOS Safety-of-life (SoL) Service is intended for transport applications in different domains (and currently in use by Aviation) where lives could be endangered if the performance of the navigation system is degraded below specific accuracy limits without giving notice in the specified time to alert. This requires that the relevant authority of the particular transport domain determines specific requirements for the navigation service based on the needs of that domain.

Even if the main objective of the SBAS systems is the civil aviation community, the advantages provided by this technology are very useful to users from other domains. In this sense, a new EGNOS service for maritime is currently under development with the objective to complement the existing maritime radionavigation systems (e.g. DGNSS) in the European region for enhanced accuracy and integrity information where there is no backup infrastructure or in poorly covered environments.
\end{abstract}

One of the steps needed for the development of this new EGNOS maritime service is the definition of a minimum set of recommendations for receiver manufacturers to provide them with a clear view on how to design their SBAS receivers to be compliant with the requirements defined for such a service. For that, EC, GSA, ESA and ESSP SAS have been working together since 2016 to develop guidelines for manufacturers for the implementation of SBAS in shipborne receiver. These guidelines, developed in the frame of the SBAS Working Group created in the Special Committee (SC) 104 on Differential Global Navigation Satellite Systems (DGNSS) of Radio Technical Commission for Maritime Services (RTCM), define a minimum set SBAS messages to be compliant with the International Maritime Organization (IMO) Resolution A.1046 and additionally provide a test specifications.

This paper presents a summary of these SBAS guidelines as well as the preliminary list of tests that must be fulfilled to be compliant. Additionally, a preliminary performance assessment of the EGNOS maritime service based on IMO Res. A.1046 (27) for a 24-months period during 2016, 2017 and 2018 is presented. The performance parameters are calculated using real data to show what level of performance was attained by 
EGNOS. The assessment was done using both EGNOS ground monitoring stations (RIMS) and fault-free receivers, based on these guidelines, fed with actual data. The performance is shown for each performance parameter defined in the IMO Res. A.1046 (27) and for navigation in Ocean Waters and coastal waters, harbour entrances and harbour approaches. The paper also includes Service Coverage maps representing where EGNOS maritime service based on IMO Res. A.1046 (27) is fulfilling the requirements.

Furthermore, GSA and ESSP, with the collaboration of The Norwegian Coastal Administration and Hurtigruten Cruises, carried out a GNSS data collection campaign of 10 days along the Norwegian coast with a trajectory through Trondheim to Kirkenes and Kirkenes to Bergen in February 2018. The aim of this data campaign was to assess EGNOS performance at user level in the maritime domain at high latitudes in Europe. The data campaign includes the navigation outside the MT27 region defined in EGNOS at that moment $\left(70^{\circ} \mathrm{N}\right)$. A performance assessment of EGNOS using some commercial receivers and a software receiver in line with the SBAS guidelines will be presented, showing the observed accuracy and availably results of the EGNOS solution.

Keywords - SBAS, EGNOS

\section{INDRUCTION}

EGNOS is the Satellite Based Augmentation System (SBAS) over Europe. EGNOS is a positioning and timing service currently designed to augment the GPS navigation system constellations by broadcasting additional free-of-charge signals from geostationary (GEO) satellites.

EGNOS is widely used in aviation over Europe, which is a safety critical application. In February 2019, 196 airports have at least one EGNOS based procedure for landing and/or taking off. Apart from providing enhanced accuracy with respect to GPS standalone, EGNOS provides Integrity for Safety of Life applications. Some events of the GNSS system may lead to positioning errors that are greater than the ones allowed in certain safety critical applications. EGNOS provides Integrity by detecting and removing those system errors, and by alerting the user in due time when such errors occur and cannot be discarded. EGNOS is also used in a wide range of applications (such as maritime, agriculture and surveying) that benefits the enhanced position solution with respect to GPS standalone solution.

In the maritime domain, the use of SBAS for navigation has clear benefits. In fact, most of maritime receivers are SBAS compatible, demonstrating that the use of this technology is ready to be implemented, being only limited by the lack of a clear standardisation framework. As a first step to solve this problem, the International Maritime Organisation (IMO) published the IMO resolution A.1046 (27) [IMO Res 1046, 2011], which establishes the requirements that a certain radionavigation system shall fulfil to be recognized by IMO as a component of the Worldwide Radionavigation Systems. This means that the system is recognized to be able to provide adequate position information within its service area and that the carriage of receiving equipment for use with the system satisfies the relevant requirements of the 1974 SOLAS Convention. IMO has developed a number of shipborne receivers' performance standards covering the use of GNSS, such as GPS [IMO Res. MSC.112, 2000] and DGPS [IMO Res. MSC.114, 2000]. Accordingly the International Electrotechnical Commission (IEC) produced several test specifications to accompany these IMO performance standards, which are covered in "Maritime navigation and radiocommunication equipment and systems - Global navigation satellite systems (GNSS)" for GPS, [IEC 61108-1, 2003], and DGNSS, [IEC 61108-4, 2004]. However, although IEC 61108-4 allows the use of EGNOS, there is no guidance for its implementation or standards to support the use of the SBAS system for maritime applications.

Because of that, EC (EGNOS owner), GSA (EGNOS program manager), ESA (EGNOS design agency) and ESSP (EGNOS service provider) are working in close collaboration to support the standardisation of SBAS in maritime receivers considering that EGNOS meets the requirements for general maritime navigation established in the IMO (International Maritime Organization) resolution A.1046 (27). 
A guidelines document for manufacturers for the development of SBAS receivers for the maritime domain ([Draft SBAS Guidelines, 2017]) is under development. These guidelines, together with a set of test specifications, are being developed in the frame of the SBAS Working Group created in the Special Committee (SC) 104 on Differential Global Navigation Satellite Systems (DGNSS) of Radio Technical Commission for Maritime Services (RTCM). A first draft was already distributed to some maritime receiver manufactures, which presents recommendations on the set of SBAS messages to be processed to be compliant with the IMO Resolution A.1046 (27), as well as the system alerts applicable. It also includes the tests specifications to be passed. A summary of these guidelines will be presented in this paper.

Furthermore, the paper will present the IMO Res. A.1046 (27) service performance parameters and the applicability of EGNOS in the maritime sector. All IMO Res. A.1046 (27) parameters will be addressed in this paper: Signal Availability, Horizontal Accuracy 95\%, Service Continuity, Position Update Rate, Time To Alarm and Service Coverage.

GSA and ESSP, with the collaboration of The Norwegian Coastal Administration and Hurtigruten Cruises, have carried out a GNSS data collection campaign of 10 days along the Norwegian coast with the aim of assessing EGNOS performance at user level in the maritime domain at high latitudes in Europe. SBAS performance results of this data campaign will be presented to show the added value of EGNOS in maritime applications. Several SBAS compatible receiver and a Software Receiver (compliant with the developed SBAS guidelines) will be used to analyse the performance results in terms of accuracy and availability.

Finally, the performances obtained with the receiver will be compared with the requirements of the IMO Resolution A.1046 (27) to demonstrate the feasibility of EGNOS for some maritime applications. This resolution is particularly important as it states the minimum operational requirements that the maritime community requires from any navigation system in order to be accepted.

The work presented in this paper, which gathers the SBAS guidelines document and the SBAS performance results obtained in this data campaign in Norway, are intended to support the integration of SBAS in the standardization process for maritime navigation and radiocommunication equipment and systems.

\section{SBAS GUIDELINES}

The architecture of EGNOS consists of a set of reference monitoring stations (with a well-known position) to receive GNSS constellation signals that will be processed in data processing centres to obtain some estimations of these errors that are also applicable to the users (i.e. ionospheric errors, satellite position/clock errors, etc.) as shown in Figure 1. Once these estimations are computed, they are transmitted in the form of "differential corrections" by means of at least two SBAS Geostationary satellites, which covers Europe.

In consequence, vessels can receive these SBAS corrections from SBAS GEO satellites and apply them in order to obtain an enhanced navigation position with respect to GPS standalone. In addition, vessels could get advantage of the Integrity provided by SBAS, which warn users under situations that could impact the positioning error.

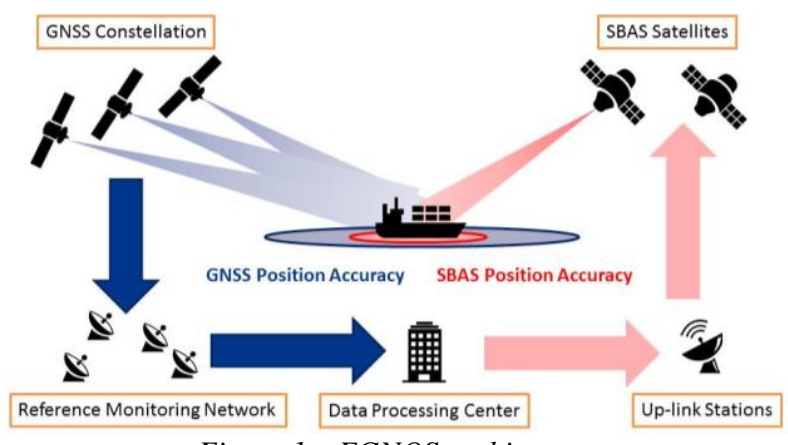

Figure 1-EGNOS architecture. 
EGNOS corrections are composed of a number of different Message Types (MT). The format and detailed information on the content and their use for SBAS certified receivers for aviation are given in [ICAO SARPs, 2018] and [DO-229, 2016].

Among those SBAS messages, the SBAS guidelines document proposes the use of a set of Message Types to fulfil IMO Res A.1046(27) performance requirements using the SBAS SiS corrections and integrity data. Summarising, a maritime receiver is expected to:

- Consider the reception of Message Type 0, it indicates that SBAS information broadcast by that GEO is not adequate for safety applications. The reception of a Message type 0 will result in the cessation of the use of that SBAS GEO for all safety applications.

- Decode and apply fast corrections information broadcast through Message Types 2 to 5 and 24 corresponding to satellites selected by Message Type 1. The equipment shall decode the UDREI (UDRE Indicator) field of Message Types 2 to 5, 24, and 6 to determine if the satellite corrections can be applied, if the satellite is not monitored or if a failure of a specific satellite is indicated by the system. Every alert condition is sent four times in four seconds.

- Decode and apply long-term satellite error corrections broadcast though Message Types 24 and 25.

- Decode and apply ionospheric corrections if available broadcast through Message Type 26 for ionospheric grid points defined by Message Type 18. The equipment shall decode the GIVEI (GIVE Indicator) field of Message Types 26 to determine if the ionospheric correction for that Ionospheric Pierce Point (IPP) can be applied, if it is not monitored or if a failure of a specific IPP is indicated by the system.

- Apply tropospheric error corrections.

- Time-out intervals for the validity of the messages as defined in the [Draft SBAS Guidelines, 2017] and [DO-229, 2016]

- Consider almanac data from SBAS satellites broadcast through Message Type 17.

- Use the augmentation satellite ranging function if available broadcast through Message Type 9 (currently not available in EGNOS).

Once all corrections have been considered, the equipment shall compute its three-dimensional position, velocity and time (PVT) using a linearized, weighted least-squares solution as defined in [DO-229, 2016].

In case the equipment computes sigma errors at pseudo-range level, for example for error ellipse estimation, the SBAS maritime receiver are recommended to:

- Process Message Types 10, 27 and 28 for the computation of the variance of fast and long term corrections residuals.

Moreover, the SBAS guidelines also includes the tests that SBAS maritime receiver manufacturers can perform to verify that the receiver is complaint with the requirements at system level, with the Signal-In-Space RF characteristics and with the SBAS messages processing indicated in that document. The proposed set of tests is:

- Static accuracy

- Static accuracy with angular movement of the antenna

- Dynamic accuracy

- Static availability

- Static continuity

- SBAS Navigation warnings and status indications

- Use of SBAS messages

- SBAS data input

- Sensitivity and dynamic range

- SBAS GEO switching 
Additionally, the definition of some checks at receiver level to improve integrity and guarantee availability performances is currently ongoing. For detailed information on the added value of the specific messages recommended, on the particularities of the way SBAS messages shall be processed and its interrelations, please contact ESSP to request further information about the development of the SBAS Guidelines document.

\section{A. EGNOS 1046 SERVICE PERFORMANCE PARAMETERS AND PRELIMINARY ASSESSMENT}

This section presents the approach that has been devised by GSA and ESSP for the applicability of EGNOS in the maritime sector. An approach similar to the one in [IALA Guideline No. 1112, 2015] which defines how to calculate the performance parameters for DGNSS is applied.

All IMO Res. A.1046 (27) parameters are addressed below: Signal Availability, Horizontal Accuracy 95\%, Service Continuity, Position Update Rate, Time To Alarm and Service Coverage. Additionally, [IALA Guidelines No.1112, 2015] introduces one additional parameter (Service Availability) and adapts the set of service performance parameters so that they account for the specific characteristics of the augmentation. For EGNOS, the same approach was followed.

\section{B. SIGNAL AVAILABILITY}

Signal availability refers to the percentage of time during which reliable information is provided by the system within the specified area of service. Thus, EGNOS 1046 Signal Availability assesses the percentage of time the EGNOS SiS is provided by the GEOs according to messages that can be processed by an SBAS receiver aligned with the receiver guidelines.

EGNOS broadcasts through two operational GEO satellites. This redundancy will benefit EGNOS 1046 receivers capable of instantaneous GEO switching and therefore, signal availability has to be calculated as the combined signal availability of both operational EGNOS GEOs. EGNOS 1046 signal availability reaches the same value in the intersection area of both EGNOS GEOs footprint (Figure 2).

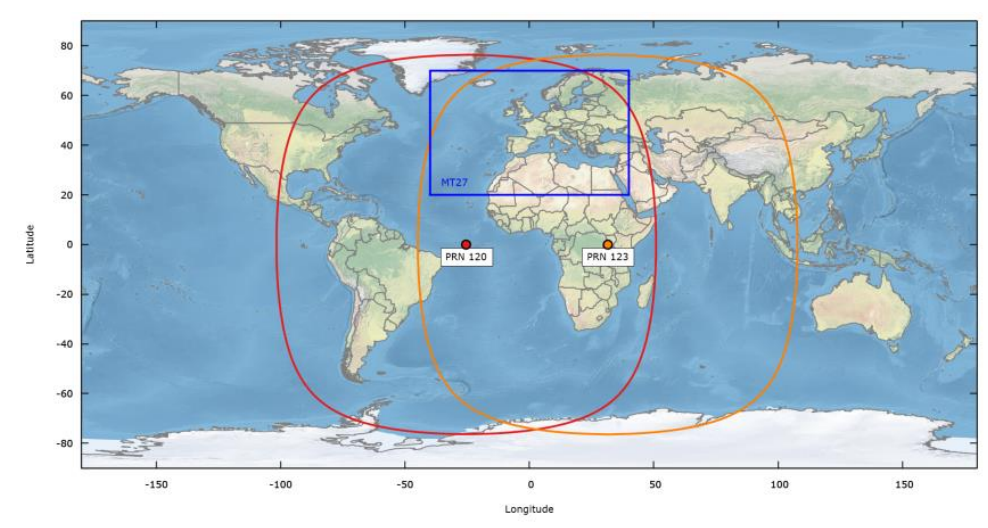




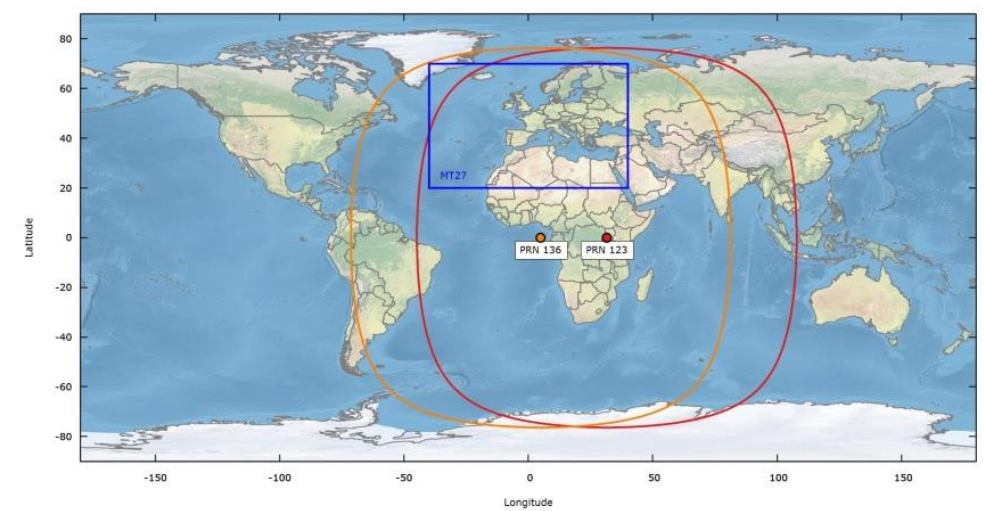

Figure 2 - Operational EGNOS GEOs footprint from October 2016 to August 2018 (top) and from August 2018 onwards (bottom).

From October 2016 to September 2018, at least one of the EGNOS GEOs in the operational configuration broadcast a valid EGNOS SiS during 100\% of the time (PRN 120/136 \& PRN123), which means that the Signal Availability reached $100 \%$ meeting the IMO requirement of $99.8 \%$.

\section{HORIZONTAL ACCURACY 95\%}

Horizontal Accuracy is the 95\% percentile of the Horizontal Position Error (HPE) distribution. HPE is the $2 \mathrm{D}$ radial error of the instantaneous measured position respect to the true instantaneous position. The following table shows the Horizontal Accuracy 95\% values in meters in EGNOS stations (Table 1):

Table 1-Horizontal accuracy 95\% from October 2016 to September 2018.

\begin{tabular}{|c|c|c|c|}
\hline Station & $\begin{array}{c}\text { Horizontal Accuracy } \\
95 \%(m)\end{array}$ & Station & $\begin{array}{c}\text { Horizontal Accuracy } \\
95 \%(\mathrm{~m})\end{array}$ \\
\hline Aalborg & 0.8 & Santiago de C. & 0.8 \\
\hline Berlin & 0.8 & Sofia & 1.0 \\
\hline Catania & 0.7 & Gävle & 0.7 \\
\hline Cork & 0.8 & Toulouse & 0.7 \\
\hline Warsaw & 0.9 & Trondheim & 0.7 \\
\hline Djerba & 0.8 & Tromsoe & 0.9 \\
\hline Egilsstadir & 0.7 & Zürich & 0.8 \\
\hline Glasgow & 0.9 & Jan Mayen & 1.2 \\
\hline Lisbon & 0.9 & Athens & 0.7 \\
\hline Swanwick & 1.0 & Agadir & 0.9 \\
\hline Madeira & 0.8 & Canary Island & 1.1 \\
\hline Málaga & 0.7 & La Palma Island & 1.0 \\
\hline Kirkenes & 0.8 & Azores & 2.8 \\
\hline Palma de Mallorca & 0.6 & Longyearbyen & 2.1 \\
\hline Reykjavik & 0.9 & Abu Simbel & 2.5 \\
\hline Roma & 0.7 & Alexandria & 1.3 \\
\hline Lappeenranta & 0.7 & Golbasi & 0.9 \\
\hline
\end{tabular}

The values above represent the Horizontal Accuracy 95\% for those epochs when a position solution based on EGNOS was available in the station. All stations meet the IMO requirements of $10 \mathrm{~m} 95 \%$ for "Harbour entrances, Harbour approaches and Coastal Waters" surrounding Europe and IMO requirements of 100m 95\% for "Ocean Waters". 


\section{SERVICE AVAILABILITY}

Performance parameters in Table 1 come from IMO Res. A.1046 (27). As a radionavigation aid, EGNOS has a particularity: EGNOS GEO satellites broadcast messages over the GEOs footprint (Figure 2) but EGNOS performance is not the same in the whole EGNOS GEO footprint. In some areas, where EGNOS SiS is received, EGNOS 1046 receivers will not be able to compute a position solution based on EGNOS (e.g. south of Africa). This happens because EGNOS messages include ionospheric corrections only for a region around Europe (Figure 3) and, for a specific epoch, only for a subset of GPS satellites. This subset of satellites is the specific subset in view from Europe and the surrounding area (Figure 4):

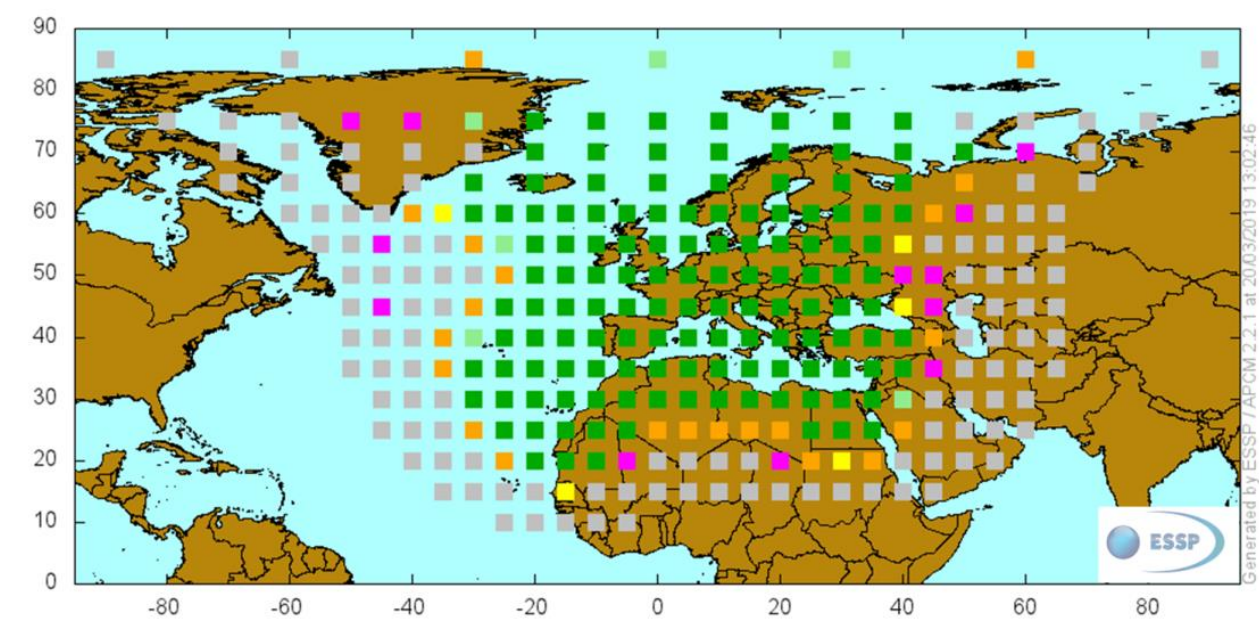

Figure 3-EGNOS ionospheric grid points (IGP) for ionospheric differential corrections and integrity information.

Figure 3 presents in green IGPs monitored which means the differential corrections and integrity information are available for that IGP and can be used by EGNOS users and in a different color IGPs partially monitored or not valid. A similar situation happens for each GPS satellite. Figure 4 presents in green GPS satellites monitored by EGNOS during a period of two hours and in black GPS satellites which were not monitored during that period of time:

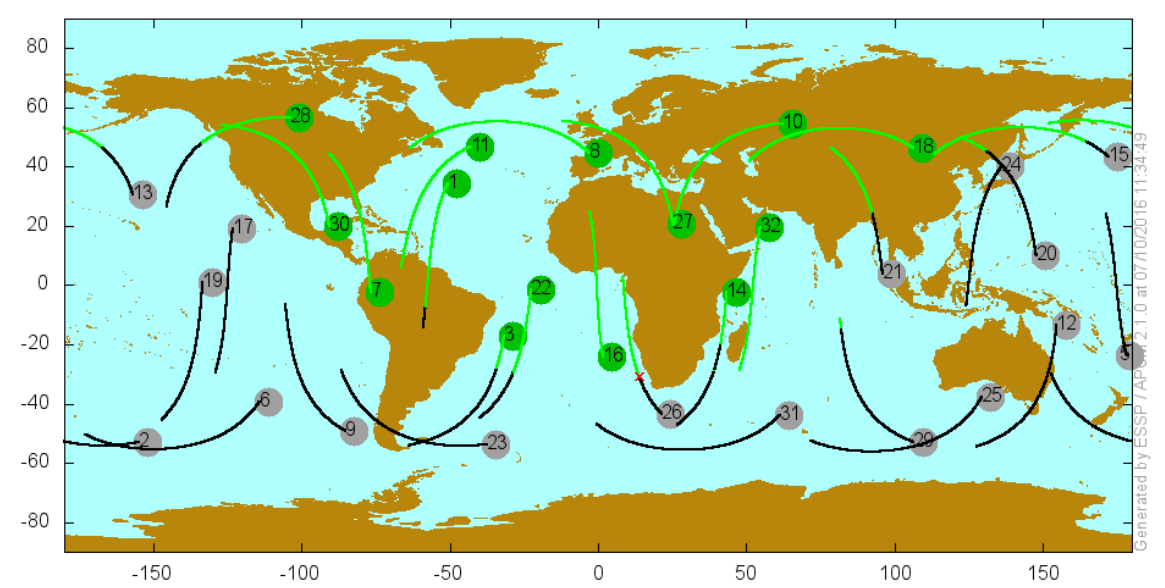

Figure 4-GPS satellites monitored by EGNOS (in green) during a period of two hours.

A GPS satellite is monitored if EGNOS differential corrections and integrity information is available for that GPS satellite. Then, this GPS satellite can be used for the EGNOS position solution. As a result, users in Europe and the surrounding area can use EGNOS differential corrections and integrity information to improve their positioning. 
In order to evaluate when EGNOS can be used to calculate a position solution, it is used the EGNOS Service Availability concept. This is defined as the percentage of time a position calculated using EGNOS for maritime is available in a specific location. EGNOS 1046 Service Availability shall be calculated considering receivers are able to do instantaneous GEO switching. For Service Availability, which is not an IMO requirement, it is proposed a target value of $99.8 \%$ that will ensure very high quality service. Note that the lack of Signal Availability is one of the events affecting the Service Availability. According to this definition, Figure 5 shows the Service Availability from October 2016 to September 2018.

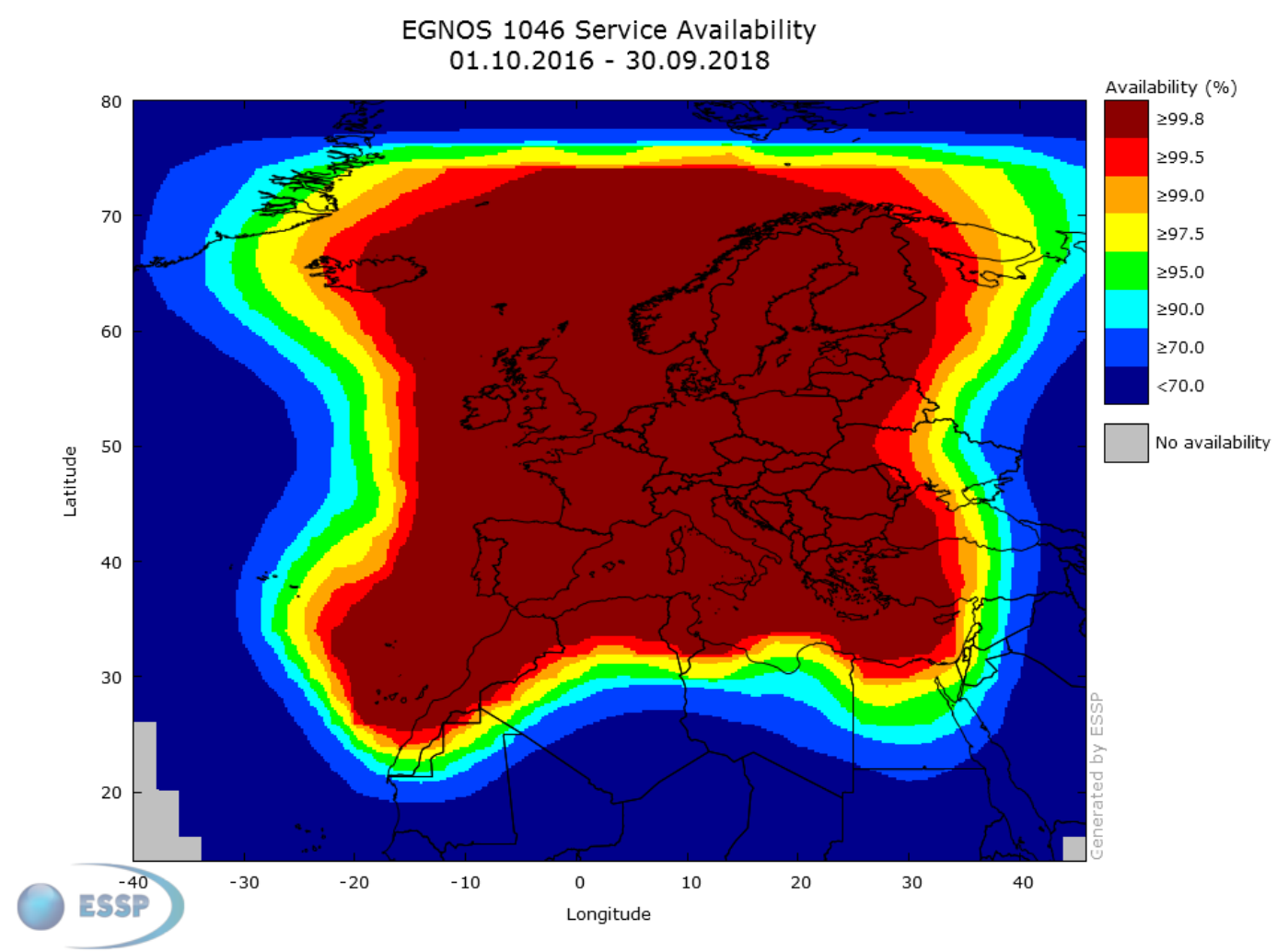

Figure 5 - EGNOS 1046 Service Availability from October 2016 to September 2018.

\section{E. SERVICE CONTINUITY}

The Service continuity is the probability that a lack of position-fixing event will start during the Continuity Time Interval (CTI), which is defined to be 15 min [IALA Guideline No. 1112, 2015]. EGNOS 1046 Service Continuity is closely linked to Service Availability. Every transition from a service available to unavailable is a service continuity event. Service Continuity is calculated using combined GEOs. Equation [1] shows how EGNOS 1046 Service Continuity is calculated:

$$
\text { Service Continuity }=1-\frac{C T I}{M T B F}
$$

Where CTI is $15 \mathrm{~min}$ and MTBF is the "Mean Time Between Failures", as measured by the service provider [IALA Guideline No. 1112, 2015] (e.g. over two years). The $99.97 \%$ over $15 \mathrm{~min}$ requirement is a challenging requirement. EGNOS shall present on average only one Service Continuity event per month.

EGNOS SiS monitoring from October 2016 to September 2018, reports the following percentages of Service Continuity for "Harbour entrances, Harbour approaches and Coastal Waters" surrounding Europe. For these 
applications, the area to be considered is up to $12 \mathrm{~nm}$ from the coast line (territorial waters), where the continuity requirement applies. Note there is no IMO continuity requirement for "Ocean Waters" (Figure 6):

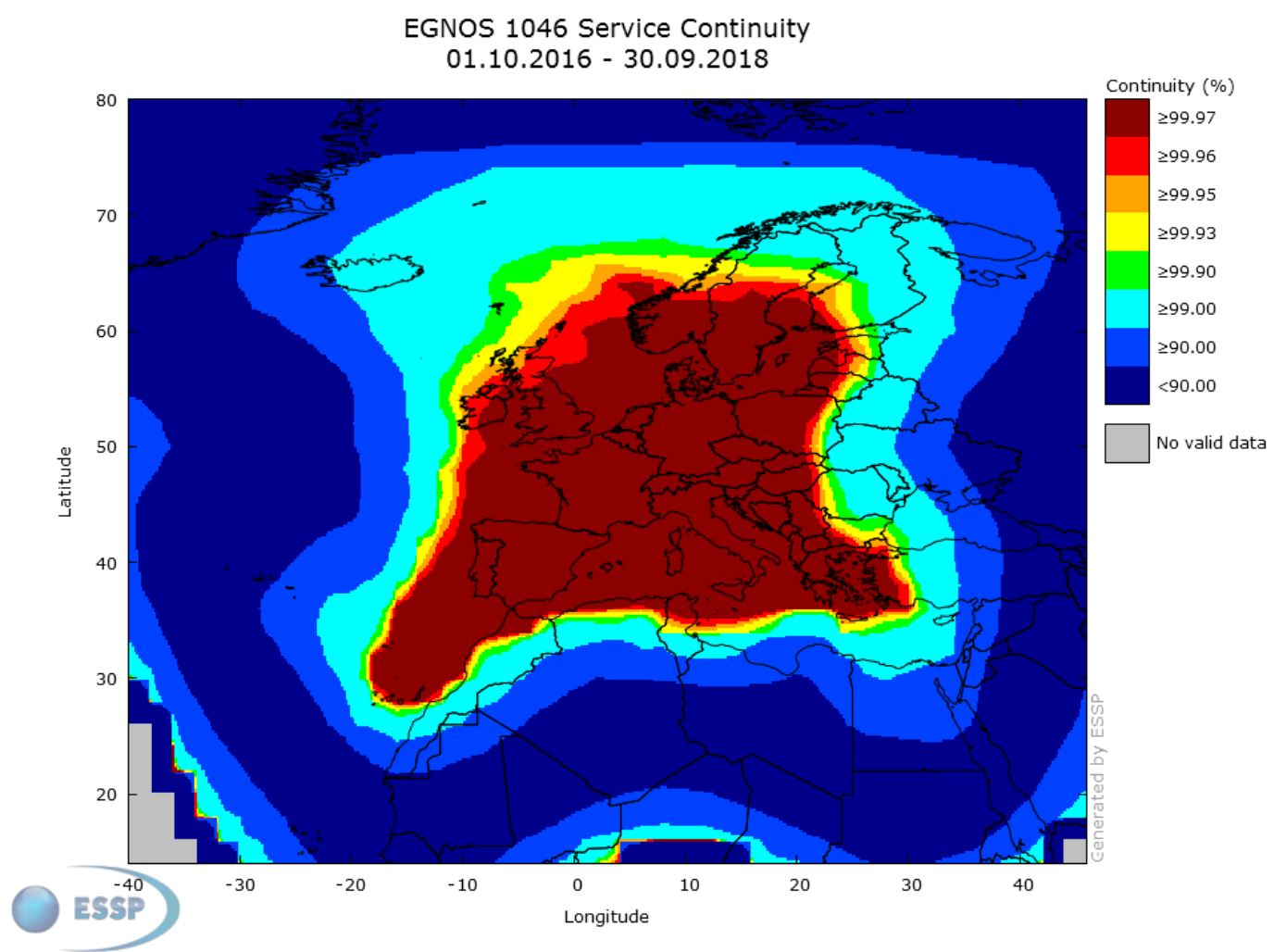

Figure 6-EGNOS 1046 Service Continuity from October 2016 to September 2018 for harbour entrances/approaches and coastal waters.

The reduced area meeting the Service Continuity IMO requirement (99.97\% over $15 \mathrm{~min})$ over some areas is due to events that affected EGNOS PVT either on a major part or on the whole service area. Some occurrences of events affecting limited areas were observed over the analysed period reducing drastically the Service Continuity area reaching the $99.97 \%$ over $15 \mathrm{~min}$.; in particular, the coverage in the northern area was impacted several days (e.g. 08/09 in 2017 and 23/03 and 26/08 in 2018) by some ionospheric events.

\section{F. POSITION UPDATE RATE}

EGNOS 1046 receivers must be designed to meet the 2 seconds update rate required by IMO Resolution A.1046 (27). The compliance to this parameter shall be demonstrated by the receiver/equipment manufacturers. EGNOS 1046 receivers using a subset of SBAS [4] messages broadcast by EGNOS, are expected to be capable of an update rate of 1 second of the computed position.

\section{G. TIME TO ALARM}

The requirement in IMO Resolution A.1046 (27) is 10 seconds for "Harbour entrances, Harbour approaches and Coastal Waters". EGNOS design ensures timely integrity alarms to GPS satellites for pseudorange and ionospheric corrections in 6 seconds. Whenever there is an integrity alarm in a satellite or IGP, EGNOS sets that satellite or IGP to "do not use". No Time to Alarm requirement for "Ocean Waters" is set on EGNOS 1046. 


\section{H. SERVICE COVERAGE}

The service coverage is a designated geographical area where, taking into account the radio frequency environment, EGNOS is adequate to provide required performance throughout a phase of navigation. By "adequate" it is understood that for a specific location EGNOS 1046 meets for Ocean Waters, Harbour entrances, Harbour approaches and Coastal Waters, the 95\% Horizontal Accuracy, the Signal Availability and the Service Availability requirements.

For "Harbour entrances, Harbour approaches and Coastal Waters", performance results on Signal Availability, Service Availability and Service Continuity showed in previous sections, identify a geographical area where EGNOS meets IMO requirements. In this area (Figure 7 left) EGNOS was adequate to provide required performance throughout for the period from $1^{\text {st }}$ October 2016 to $30^{\text {th }}$ September 2018.

For "Ocean Waters", performance results on Signal Availability and Service Availability showed in previous sections identify a geographical area where EGNOS meets IMO requirements. In this area (Figure 7 right) EGNOS was adequate to provide required performance throughout for the period from $1^{\text {st }}$ October 2016 to $30^{\text {th }}$ September 2018.
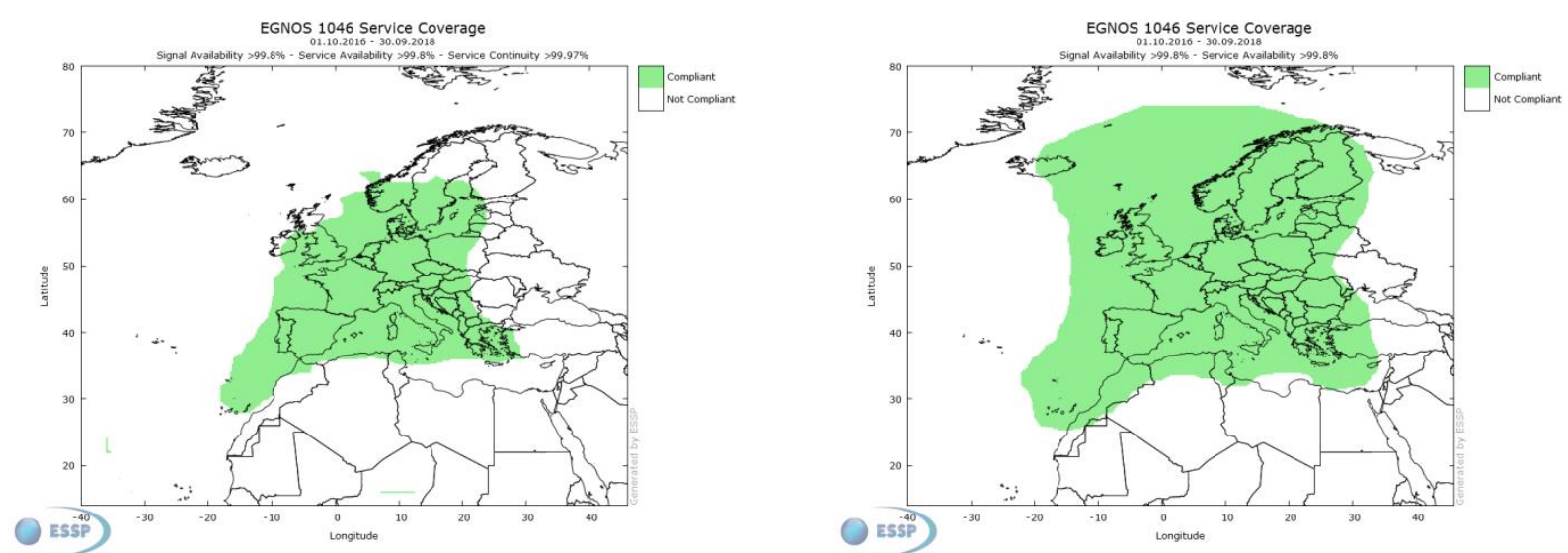

Figure 7 - EGNOS 1046 Service Coverage October 2016 to September 2018 for "Harbour entrances, Harbour approaches and Coastal Waters" (left) and "Ocean Waters" (right).

In case that EGNOS would be used as a back-up for existing IALA DGNSS services, the continuity requirement could be relaxed from $99.97 \%$ to $99.95 \%$ following the logic of overlapping services described in [IALA Guideline No. 1112, 2015]. Figure 8 shows the impact in the service coverage area depending on the continuity requirement. 


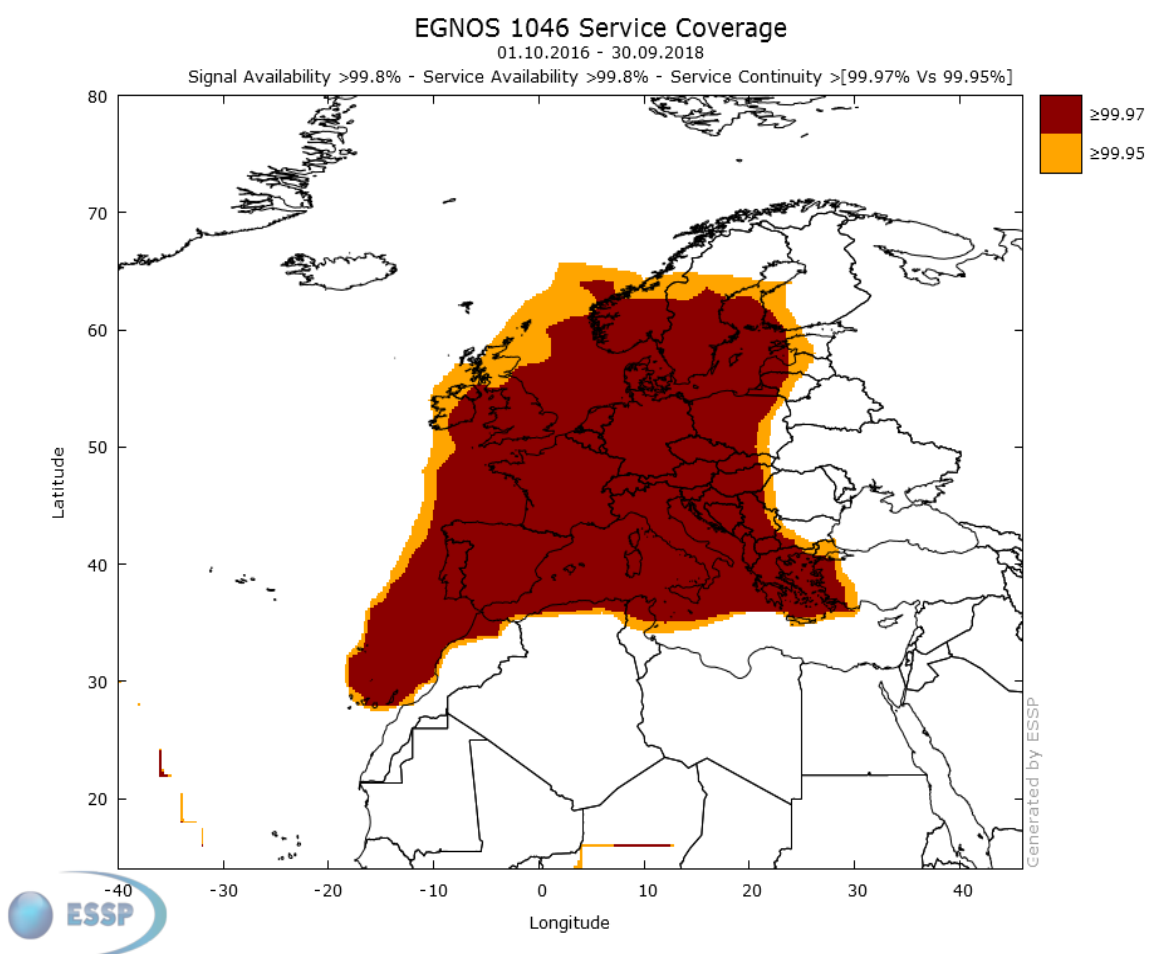

Figure 8-EGNOS 1046 Service Coverage for Harbour entrances, Harbour approaches and Coastal Waters (with Service Continuity >99.97\% vs. with Service Continuity >99.95\%) October 2016 to September 2018.

Service Coverage maps presented in this work are preliminary results. Taking into account several Service Continuity events that arose during the period of analysis, both "Harbour entrances, Harbour approaches and Coastal Waters" the Service Coverage maps are considered sufficiently good.

\section{Data Campaign}

In order to assess the EGNOS performance at user level in the maritime domain at high latitudes in Europe, a data campaign was carried out along the Norwegian Coast on February and March 2018. Figure 9 shows the route followed by the vessel along the mission. 


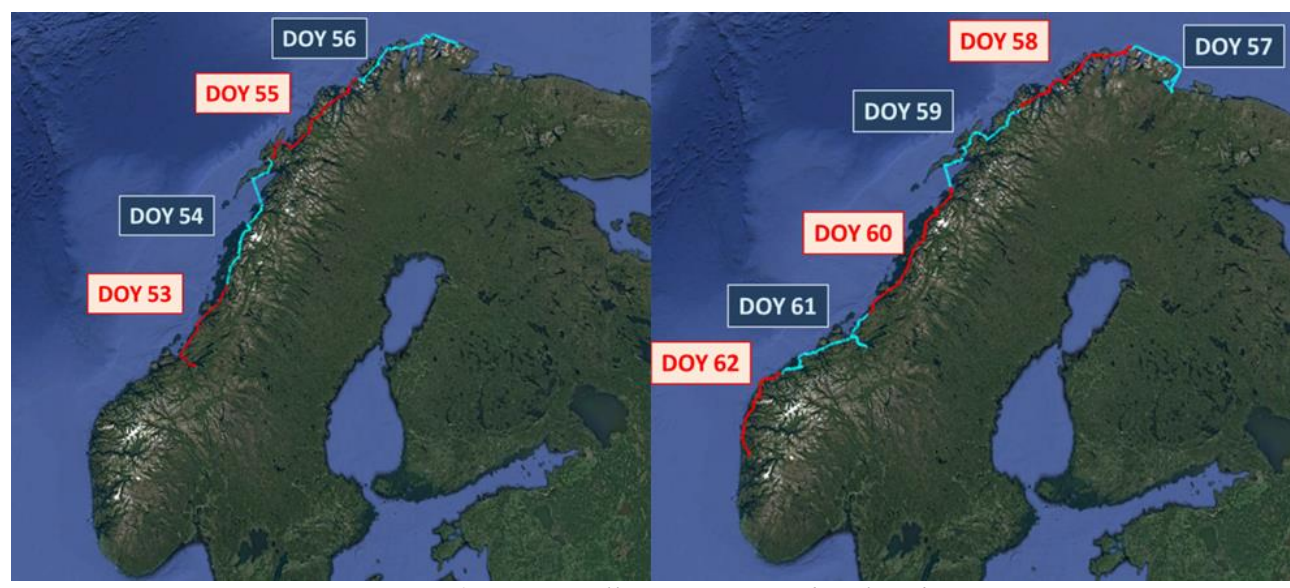

Figure 9 -Data collection geographical path.

The EGNOS receiver installed in the vessel directly reported the PVT solution based on EGNOS. Additionally, this receiver provided dual frequency and multi constellation observations which were injected offline to Precise Point Positioning (PPP) algorithms in order to compute the precise position of the vessel at any time. Besides, these dual frequency observations were also injected in the Software receiver, gLAB (http://www.gage.upc.edu/gLAB) to compute the EGNOS performance in line with the Guidelines for Manufacturers for the Implementation of SBAS in Shipborne Receivers. It is noted that gLAB was modified following the SBAS guidelines and that it passed the test specification provided in those guidelines).

Finally, the L1 RF data recorded by LabSat 3 was injected in both Maritime receiver with EGNOS capability and a GPS standalone receiver to obtain EGNOS PVT solution and GPS standalone PVT solution, respectively. The aim of that is to provide a comparison between GPS and EGNOS performance in that region.

The EGNOS monitoring information shows that the EGNOS signal in space (SIS) availability was $\mathbf{1 0 0 \%}$ during the data campaign period. The strength of the EGNOS signal along the route is also checked. Figure 10 shows the temporal evolution of the carrier-to-noise ratio (C/N0) values for the three EGNOS GEO satellites. During the data collection, PRN 120 and 123 were operational, whereas PRN 136 was set in test mode. The analysis depicts that PRN 123 and PRN 136 signal were received with a high strength. PRN 120 was received with lower $\mathrm{C} / \mathrm{N} 0$ especially during the days that the vessel was in the North of Norway (doy from 55 to 59), because it was observed from low elevations, i.e. in the border of the PRN 120 coverage area.

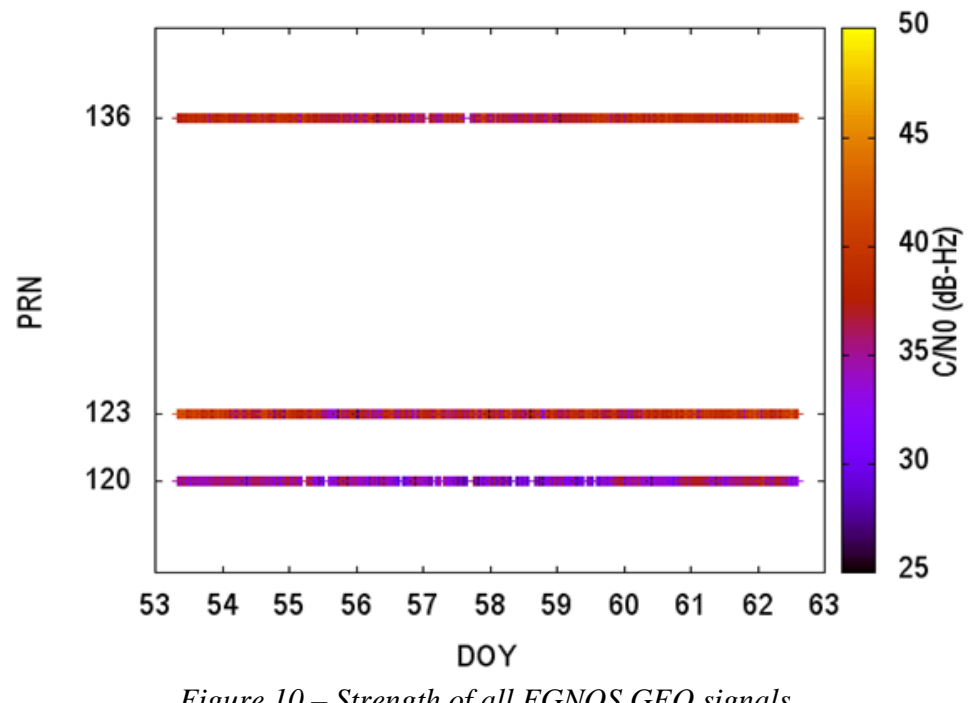

Figure 10 - Strength of all EGNOS GEO signals. 
Although the EGNOS SIS availability was $100 \%$, some data gaps were detected, that is, lack of EGNOS signal tracking by the receiver, linked to the orography of the region, not to the EGNOS system. The EGNOS user support website (https://egnos-user-support.essp-sas.eu/new_egnos_ops/resources-tools/egnosvisibility-maps) shows the areas over Europe where there is no visibility of one or both EGNOS operational geostationary satellites (PRN 120 and PRN 123 by the time of the data campaign).

The impact of the orography in the availability of the EGNOS signal received by the receiver is greater the lower the elevation angle of the SBAS GEO satellite is. Figure 11 depicts the EGNOS signal availability in the EGNOS receiver as function of the latitude. This figure confirms that the impact of the orography is greater according the latitude is increasing (or the SBAS GEO satellite elevation angle decreased).

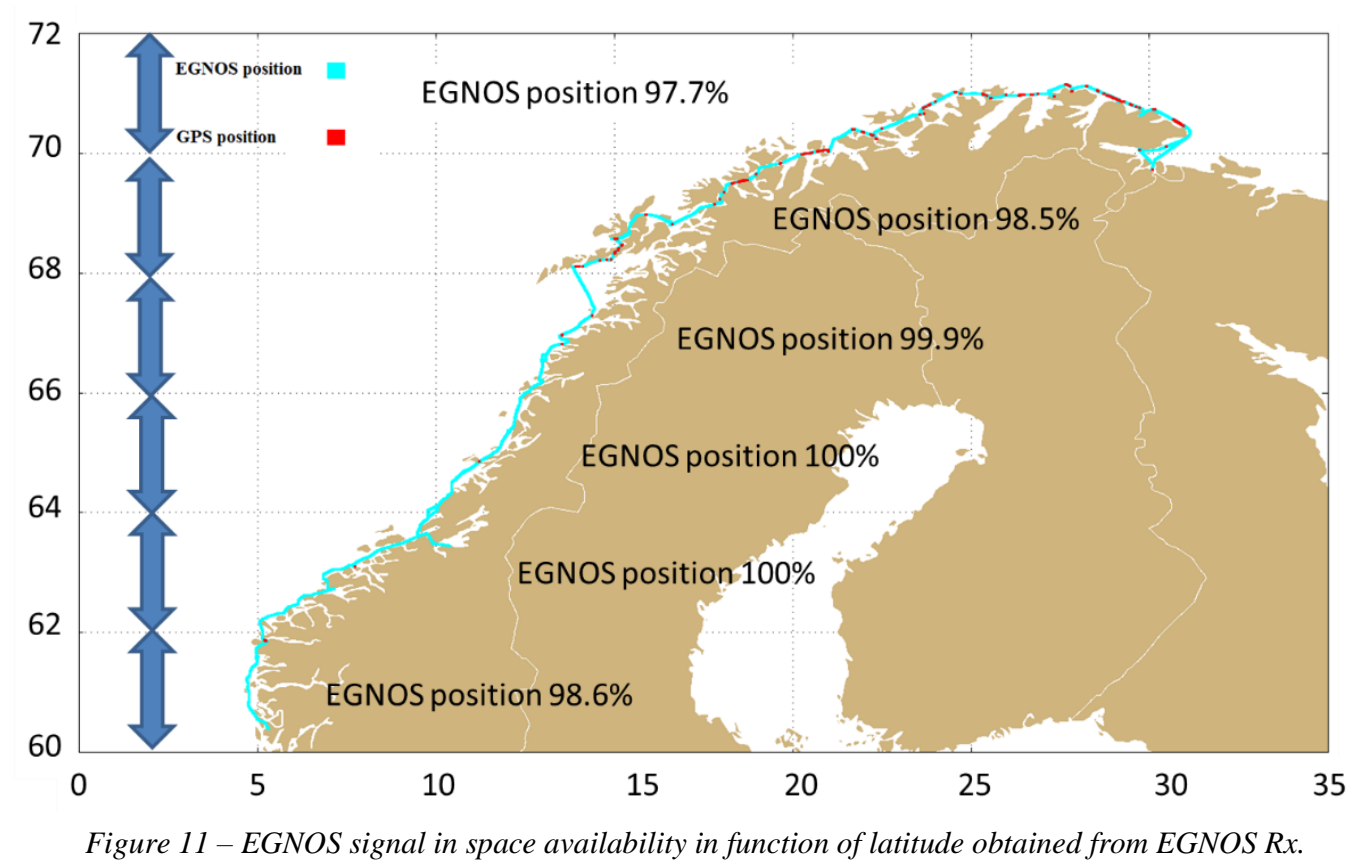

As example, in the latitudes from 60 to 62 degrees the EGNOS position is only $98.6 \%$ because the vessel is located very close to a mountain that obstructs the GEO satellite visibility, as it is seen in Figure 12. 


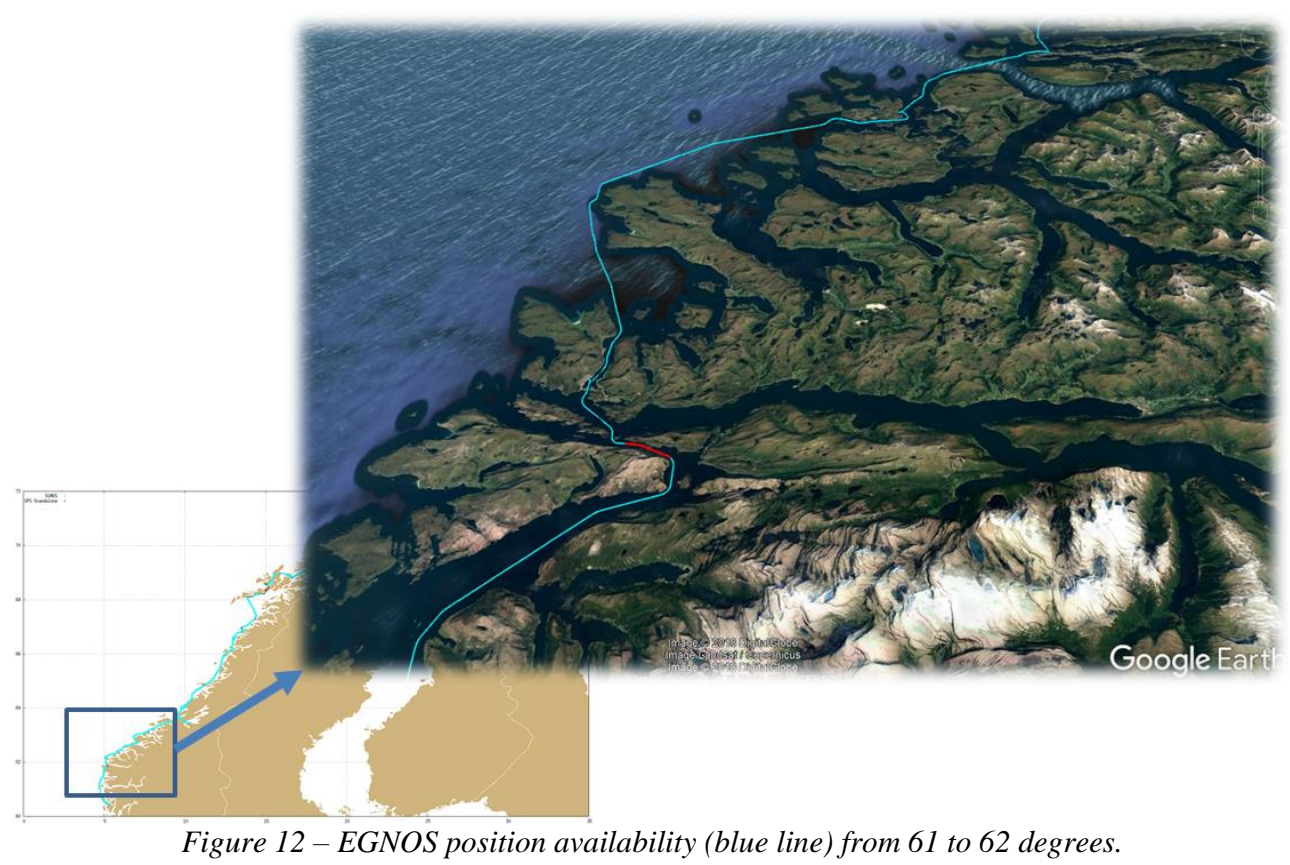

The daily Horizontal Position Error (HPE) 95-th percentile values are computed for the four datasets: EGNOS Receiver, Maritime Receiver with EGNOS capability, gLAB (Software Receiver aligned with SBAS guidelines) and GPS Standalone Receiver. Table 2 presents the daily HPE 95-th percentile values confirming that gLAB (EGNOS software receiver) provides the best (the lowest HPE values) accuracy performances with values about $1 \mathrm{~m}$. It is remarked that this software receiver is aligned with the SBAS guidelines. It is also observed that the Maritime Receiver with EGNOS capability provides HPE 95-th percentile values very close to those obtained from gLAB. On the other hand, the EGNOS receiver provides a very good HPE 95-th percentile values, about $1.2 \mathrm{~m}$, slightly greater than those obtained from EGNOS SW receiver and the Maritime receiver with EGNOS capability. Considering that gLAB is a Software tool in post-processing, it does not have time processing restrictions like the real-time receiver. In addition, other algorithms considerations (such as smoothing filter using phase, Kalman filter, least squares applicability, dynamic conditions configuration, EGNOS message type processing ...) could provide slightly different position results. This is also applicable to the Maritime receiver with EGNOS capability: algorithms assumptions are oriented to maritime environment, so better performances than EGNOS Receiver could be expected. As expected, the GPS standalone receiver provides the worst HPE 95-th percentile values, typically greater than $2 \mathrm{~m}$. These values show that the receiver that used EGNOS provides a more accurate position during the whole period evaluated that the receiver GPS standalone.

Table 2 -.Daily HPE 95-th (meters) for SBAS receivers, $g L A B$ and GPS standalone.

\begin{tabular}{ccccc}
\hline DOY & EGNOS Rx & $\begin{array}{c}\text { Maritime } R x \text { with } \\
\text { EGNOS capability }\end{array}$ & $\begin{array}{c}\text { gLAB } \\
\text { (SW Rx) }\end{array}$ \\
\hline 53 & 1.24 & 0.98 & 1.14 & 2.08 \\
54 & 1.21 & 0.93 & 0.95 & 2.00 \\
55 & 1.41 & 1.09 & 1.03 & 2.09 \\
56 & 1.30 & 0.98 & 0.92 & 1.87 \\
57 & 1.23 & 0.99 & 0.97 & 1.87 \\
58 & 1.44 & 1.13 & 0.96 & 2.00 \\
59 & 1.23 & 1.07 & 0.89 & 2.05 \\
60 & 1.18 & 0.83 & 0.89 & 2.05 \\
61 & 1.14 & 0.88 & 0.80 & 2.36 \\
\hline
\end{tabular}




\section{Conclusions}

This paper has presented a summary of the document providing draft Guidelines to Receiver Manufacturers for the development of SBAS in shipborne receivers in compliance with the International Maritime Organization (IMO) Resolution A.1046 (27).

EGNOS performances were assessed against performance requirements defined in IMO Resolution A.1046(27). This assessment has shown that current EGNOS system is compliant with Signal Availability, Horizontal Accuracy 95\%, Position Update Rate and Time to Alarm requirements. In addition, it is provided the EGNOS Service Coverage areas over Europe for "harbour entrances/approaches and coastal waters" and for "Ocean Waters" meeting the requirements of in IMO Resolution A.1046 (27) from October 2016 to September 2018.

The GNSS tool gLAB has been upgraded to be aligned with these draft SBAS guidelines in order to confirm that the expected performance requirements defined in the IMO Resolution A.1046(27) are satisfied.

From data campaign results, it is extracted that:

- The EGNOS signal in space availability was $100 \%$ during the data campaign period. But it should be noted that some data gaps were detected, that is, lack of EGNOS signal tracking by the receiver, linked to the orography of the region.

- As expected, it was also demonstrated that EGNOS provides better accuracy performance than GPS Standalone, including outside the EGNOS Service Area defined in Message Type 27. Accuracy figures are degraded between $30 \%$ and $50 \%$ in GPS standalone.

The work presented in this paper, which gathers the SBAS guidelines document and the SBAS performance results obtained in this Norway data campaign, are intended to support the integration of SBAS in the standardization process for maritime navigation and radiocommunication equipment and systems within IEC 61108 .

\section{ACKNOWLEDGMENTS}

Authors would like to express their gratitude to the Norwegian Coastal Administration for their collaboration in order to test the EGNOS performances in the Norwegian coasts and to Hurtigruten Cruises and MS Finnmarken for letting us to use its cruise ship for this GNSS data campaign.

Authors would also like to acknowledge the efforts done by EC and the GSA to continuously support the EGNOS programme.

\section{REFERENCES}

[1] Draft Guidelines for Manufacturers for the Implementation of SBAS in Shipborne Receivers Including Methods of Testing and Required Test Results, 31 July 2017

[2] ICAO Standards and Recommended Practices (SARPS) Annex10 Volume I (Radio Navigation Aids), Amendment 91, 2018

[3] IMO Resolution A.1046(27) Worldwide Radionavigation System, 20 December 2011.

[4] IMO Resolution MSC.112(73), Adoption of the revised performance standards for shipshore Global Positioning System(GPS) receiver equipment, 1 December 2000 
[5] IMO Resolution MSC.114(73), DGPS and DGLONASS maritime radio beacon receiver equipment, 1 December 2000.

[6] IMO Resolution MSC.401(95), Performance standards for multi-system shipborne radionavigation receivers, 8 June 2015.

[7] IEC 61108-1, Maritime navigation and radiocommunication equipment and systems - Global navigation satellite systems (GNSS) - Part 1: Global positioning system (GPS) - Receiver equipment - Performance standards, methods of testing and required test results, 2003.

[8] IEC 61108-4, Maritime navigation and radiocommunication equipment and systems - Global navigation satellite systems (GNSS) - Part 4: Shipborne DGPS and DGLONASS maritime radio beacon receiver equipment - Performance requirements, methods of testing and required test results, 2004.

[9] EGNOS Multimodal adoption plan, EGNOS Workshop, Athens, 2017 (https://egnos-usersupport.essp-sas.eu/new_egnos_ops/sites/default/files/workshop2017/EWS17\%20GSA\&ESSP\%20\%20EGNOS\%20Multimodal\%20Adoption\%20Plan\%202017.pdf).

[10] IALA Guideline No. 1112 On Performance and Monitoring of DGNSS Services in the Frequency Band 283.5-325KHz. Edition 1. May 2015.

[11] RTCA DO 229 Minimum Operational Performance Standards for Global Positioning System/Wide Area Augmentation System Airborne Equipment (MOPS), Revision E, 15 December 2016. 